\title{
AN UPPER BOUND ON THE ATTRACTOR DIMENSION OF A 2D TURBULENT SHEAR FLOW WITH A FREE BOUNDARY CONDITION
}

\author{
MAHDI BOUKROUCHE \\ Laboratory of Mathematics, University of Saint-Étienne, EA-3989, 23 \\ rue du Dr Paul Michelon, 42023 Saint-Étienne, France \\ E-mail: Mahdi.Boukrouche@univ-st-etienne.fr
}

\section{GRZEGORZ ŁUKASZEWICZ}

Mathematics Department, University of Warsaw, Banacha 2, 02-957 Warszawa, Poland E-mail: glukasz@mimuw.edu.pl

\begin{abstract}
We consider a free boundary problem of a two-dimensional Navier-Stokes shear flow. There exist a unique global in time solution of the considered problem as well as the global attractor for the associated semigroup. As in [1] and [2], we estimate from above the dimension of the attractor in terms of given data and the geometry of the domain of the flow. This research is motivated by a free boundary problem from lubrication theory where the domain of the flow is usually very thin and the roughness of the boundary strongly affects the flow. We show how it can enlarge the dimension of the attractor. To this end we establish a new version of the Lieb-Thirring inequality with constants depending on the geometry of the domain.
\end{abstract}

1. Introduction. We consider the problem of finite dimensionality of a strongly turbulent boundary driven flow considered in lubrication theory. The two-dimensional domain occupied by the flow is assumed to be periodic with a period cell $\Omega$ a thin (or elongated) domain along the first coordinate direction (not necessarily just a rectangle), so that the boundary $\partial \Omega$ consists of three relevant parts: the upper boundary $\Gamma_{1}$, the lower boundary $\Gamma_{2}$, and the lateral boundary parts $\Gamma_{L}$. The velocity of the fluid satisfies a free boundary condition on the upper boundary and non homogeneous boundary condition on the lower

2000 Mathematics Subject Classification: 35R35, 76F10.

Key words and phrases: Navier-Stokes equations, free boundary condition, lubrication theory, global in time solution, energy dissipation rate, dimension of global attractor, Lieb-Thirring inequality.

Research of G. Eukaszewicz was supported by Polish Government grant KBN 2 P303A 030 22 and by EA-3058 UJM-Saint-Étienne France.

The paper is in final form and no version of it will be published elsewhere. 
boundary. We show how the geometry of $\Omega$ may affect the dimension of the global attractor associated with the flow, by giving un upper bound on its dimension that depends explicitly on the geometry of $\Omega$ and on the data. Our estimate reduces to one obtained earlier in [5] or [10] when $\Omega$ becomes an elongated rectangle.

There is a quickly growing literature devoted to better and better estimates of averaged parameters and attractor dimension of a variety of flows. We mentioned in [1] a few positions which are related to our research and some other to give a larger context. Our result is a direct generalization of that in [1] and [10], see also [9].

This article is organized as follows. In section 2 we give a precise formulation of the considered problem and recall a result on existence of a unique global in time solution and the associated global attractor. Section 3 is devoted to estimating the time averaged energy dissipation rate of the flow. In section 4 we prove a new anisotropic version of the Lieb-Thirring inequality for functions defined on a non-rectangular domain. We use this inequality in section 5 to give an upper bound of the global attractor dimension in terms of data and geometry of the domain.

2. Formulation of the problem. We consider two-dimensional Navier-Stokes equations,

$$
\begin{gathered}
u_{t}-\nu \Delta u+(u . \nabla) u+\nabla p=0 \\
\operatorname{div} u=0
\end{gathered}
$$

in the channel

$$
\Omega_{\infty}=\left\{x=\left(x_{1}, x_{2}\right):-\infty<x_{1}<\infty, 0<x_{2}<h\left(x_{1}\right)\right\},
$$

where $h$ is a function, positive, smooth, and $L$-periodic in $x_{1}$. Let

$$
\Omega=\left\{x=\left(x_{1}, x_{2}\right): 0<x_{1}<L, 0<x_{2}<h\left(x_{1}\right)\right\}
$$

and $\partial \Omega=\bar{\Gamma}_{0} \cup \bar{\Gamma}_{L} \cup \bar{\Gamma}_{1}$, where $\Gamma_{0}$ and $\Gamma_{1}$ are the bottom and the top, and $\Gamma_{L}$ is the lateral part of the boundary of $\Omega$. We are interested in solutions of $(2.1)-(2.2)$ in $\Omega$ which are $L$-periodic with respect to $x_{1}$. We assume

$$
u . n=0, \quad \tau \cdot \sigma(u) \cdot n=0 \quad \text { at } \quad \Gamma_{1} ;
$$

the first condition in (2.3) is the nonpenetration boundary condition, while the second one means that the tangential component of the normal stress tensor $\sigma . n$ vanishes on $\Gamma_{1}$. The components of the stress tensor $\sigma$ are

$$
\sigma_{i j}(u)=\nu\left(\frac{\partial u_{i}}{\partial x_{j}}+\frac{\partial u_{j}}{\partial x_{i}}\right)-p \delta_{i j}, \quad 1 \leq i, j \leq 3
$$

where $\delta_{i j}$ is the Kronecker symbol. Moreover, we assume

$$
u=U_{0} e_{1}=\left(U_{0}, 0\right) \text { at } \Gamma_{0}
$$

where $U_{0}$ is a positive constant, and initial condition

$$
u(x, 0)=u_{0}(x) \text { for } \quad x \in \Omega .
$$

The problem is motivated by a flow in an infinite (rectified) channel $\Omega \times(-\infty,+\infty)$, where $\Gamma_{1} \times(-\infty,+\infty)$ represents the outer cylinder, and $\Gamma_{0} \times(-\infty,+\infty)$ represents the 
inner, rotating cylinder. In the lubrication problems the gap $h$ between cylinders is never constant. We can assume that the rectification does not change the equations as the gap between cylinders is very small with respect to their radii. When $h=$ const, and the free boundary condition (2.3) is replaced by

$$
u=0 \quad \text { on } \Gamma_{1},
$$

problem (2.1), (2.2), (2.6), (2.4), (2.5) was intensively studied in several contexts, some of them mentioned in [1] where the case $h \neq$ const and the first derivative $h^{\prime} \neq 0$ was studied. The problem we consider in this paper seems not to have been studied earlier.

In applications, suitable norms of $h^{\prime}$ may characterize some particular feature of the flow such as roughness of the surface of the outer cylinder, for example. It is expected that the more rough the outer cylinder is, the more turbulent is behavior of the flow, and the effect is the more observable the smaller is the gap between the cylinders.

These features of the flow can be deduced from our estimates of the dimension of the global attractor, depending on geometry of the domain of the flow, expressed in terms of the function $h$ and its first derivative $h^{\prime}$. In technical terms, the influence of geometry of the domain shows up in the constants appearing in the new anisotropic version of the Lieb-Thirring inequality we prove in this paper.

In our considerations we use the background flow method and homogenize the boundary condition (2.4) by defining a smooth background flow, a simple version of the Hopf construction, described in detail in section 3 .

Let

$$
u\left(x_{1}, x_{2}, t\right)=U\left(x_{2}\right) e_{1}+v\left(x_{1}, x_{2}, t\right)
$$

with

$$
U(0)=U_{0}, \quad U\left(h\left(x_{1}\right)\right)=0, \quad U^{\prime}\left(h\left(x_{1}\right)\right)=0, \quad x_{1} \in(0, L) .
$$

Then $v$ is $L$-periodic in $x_{1}$ and satisfies

$$
\begin{gathered}
v_{t}-\nu \Delta v+(v \cdot \nabla) v+U v,_{x_{1}}+(v)_{2} U^{\prime} e_{1}+\nabla p=\nu U^{\prime \prime} e_{1} \\
\operatorname{div} v=0 \\
v=0, \quad \text { on } \quad \Gamma_{0}, \quad v \cdot n=0, \quad \tau . \sigma(v) \cdot n=0 \quad \text { on } \quad \Gamma_{1}
\end{gathered}
$$

and initial condition

$$
v(x, 0)=v_{0}(x)=u_{0}(x)-U\left(x_{2}\right) e_{1} .
$$

By $(v)_{2}$ we denote the second component of $v$.

Now, we define a weak form of the homogenized problem above. To this end we need some notations. Let

$$
\begin{gathered}
\tilde{V}=\left\{v \in \mathcal{C}^{\infty}\left(\Omega_{\infty}\right)^{2}: \quad v \text { is } x_{1^{-}} \cdot L \text {-periodic, div } v=0, v_{\left.\right|_{\Gamma_{0}}}=0, \text { and } v \cdot n_{\left.\right|_{\Gamma_{1}}}=0\right\}, \\
V=\text { closure of } \tilde{V} \text { in } H^{1}(\Omega) \times H^{1}(\Omega), \\
H=\text { closure of } \tilde{V} \text { in } L^{2}(\Omega) \times L^{2}(\Omega) .
\end{gathered}
$$

We define scalar products

$$
(u, v)=\int_{\Omega} u(x) v(x) d x \quad \text { and } \quad[[u, v]]=(\nabla u, \nabla v)
$$


in $H$ and $V$, respectively, and norms

$$
[v]=(v, v)^{\frac{1}{2}} \quad \text { and } \quad[[v]]=[[v, v]]^{\frac{1}{2}} .
$$

We denote by $B$ the bilinear form defined for $(u, v) \in V \times V$ by

$$
(B(u, v), w)=((u \cdot \nabla) v, w) \quad \forall \omega \in V,
$$

and

$$
a(u, v)=\nu(\nabla u, \nabla v) .
$$

Then the natural weak formulation of the homogenized problem (2.9)-(2.12) is as follows.

Problem 2.1. Find

$$
v \in \mathcal{C}([0, T] ; H) \cap L^{2}(0, T ; V)
$$

for each $T>0$, such that

$$
\frac{d}{d t}[v(t), \Theta]+a(v(t), \Theta)+(B(v(t), v(t)), \Theta)=F(v(t), \Theta),
$$

for all $\Theta \in V$, and

$$
v(x, 0)=v_{0}(x)
$$

where

$$
F(v, \Theta)=-a(\xi, \Theta)-(B(\xi, v), \Theta)-(B(v, \xi), \Theta),
$$

and $\xi=U e_{1}$ is a suitable background flow.

We have the following existence theorem.

THEOREM 2.1. There exists a unique weak solution of problem 2.1 such that for all $\eta, T$, $0<\eta<T, v \in L^{2}\left(\eta, T ; H^{2}(\Omega)\right)$, and for each $t>0$ the map $v_{0} \mapsto v(t)$ is continuous as a map in H. Moreover, there exists a global attractor for the associated semigroup $\{S(t)\}_{t \geq 0}$ in the phase space $H$.

Proof. Cf. [10], see also [2].

In the next section we shall estimate the time averaged energy dissipation rate of the flow.

3. Energy dissipation rate estimate. The aim of this section is to estimate the time averaged energy dissipation rate per unit mass $\epsilon$ of the flow $u$, the weak solution of problem (2.1)-(2.5). We define

$$
\epsilon=\frac{\nu}{|\Omega|}\left\langle[[u]]^{2}\right\rangle=\limsup _{T \rightarrow+\infty} \frac{\nu}{|\Omega|} \frac{1}{T} \int_{0}^{T}[[u(t)]]^{2} d t .
$$

We estimate first the averaged energy dissipation rate of the homogenized flow $v$, and then use the relation, $\mathrm{cf} .(2.7)$,

$$
[[u(t)]]^{2}=[[v(t)]]^{2}+2 \int_{\Omega} U^{\prime}(v)_{1, x_{2}} d x+\int_{\Omega}\left|U^{\prime}\right|^{2} d x .
$$

To estimate the right hand side of (3.2) we use equation (2.13). Taking $\Theta=v$ in (2.13) and using the notation $B(u, v, w)$ for $(B(u, v), w)$, we obtain

$$
\frac{1}{2} \frac{d}{d t}[v]^{2}+a(v, v)+B(v, v, v)=F(v, v) .
$$


Since $v=0$ on $\Gamma_{0}$ and $v \cdot n=0$ on $\Gamma_{1}, v$ is $L$-periodic in $x_{1}$, and $\operatorname{div} v=0$, we have $B(v, v, v)=0$, and

$$
\frac{1}{2} \frac{d}{d t}[v]^{2}+\nu[[v]]^{2}=F(v, v)
$$

Integrating the above inequality in $t$ on the interval $(0, T)$, dividing by $T$, and taking limsup of both sides we estimate the averaged energy dissipation rate of $v$. First, however, we have to estimate carefully the term $F(v, v)$ on the right hand side of (3.4). By (2.14),

$$
F(v, v)=-a(\xi, v)-B(v, \xi, v) .
$$

We have

$$
|a(\xi, v)| \leq \nu[[\xi]] \cdot[[v]] \leq \nu[[\xi]]^{2}+\frac{\nu}{4}[[v]]^{2} .
$$

To estimate the last term in (3.5) we use the following lemma.

LEMMA 3.1. For any $\eta>0$ there exists a smooth extension

$$
\xi=\xi\left(x_{2}\right)=U\left(x_{2}\right) e_{1}=\left(U\left(x_{2}\right), 0\right)
$$

of the boundary condition for $u$, such that

$$
|B(v, \xi, v)| \leq \eta[[v]]^{2} \quad \text { for all } v \in V .
$$

Proof. Cf. [1], [2].

Lemma 3.2. Let $U$ be as in lemma 3.1. Then

$$
\int_{\Omega}\left|U\left(x_{2}\right)\right|^{2} d x_{1} d x_{2} \leq \frac{1}{2} L h_{0} U_{0}^{2} \varepsilon,
$$

and

$$
\int_{\Omega}\left|U^{\prime}\left(x_{2}\right)\right|^{2} d x_{1} d x_{2} \leq \frac{4 L U_{0}^{2}}{h_{0}} \frac{1}{\varepsilon} .
$$

where $h_{0}=\min _{0 \leq x_{1} \leq L} h\left(x_{1}\right)$.

Proof. Cf. [1], [2].

Let $h_{M}=\max _{0 \leq x_{1} \leq L} h\left(x_{1}\right)$, and $h_{M} / h_{0} \simeq 1$. Then we can define the Reynolds number of the flow $u$ by $R e=\left(h_{0} U_{0}\right) / \nu$.

LEMMA 3.3. If Re $\gg 1$ then the time averaged energy dissipation rate per unit mass $\epsilon(v)$ for the flow $v$ can be estimated as follows,

$$
\epsilon(v) \leq \frac{64 L}{|\Omega|} U_{0}^{3} \leq \frac{64}{h_{0}} U_{0}^{3}
$$

Finally, we can formulate the main theorem in this section.

THEOREM 3.1. For the Navier-Stokes turbulent flow u defined in section 2 with Re $>>1$ the time averaged energy dissipation rate per unit mass $\epsilon$ defined in (3.1) can be estimated as follows:

$$
\epsilon \leq C \frac{U_{0}^{3}}{h_{0}}
$$

where $C$ is a numerical constant. 
Proof. By (3.2) we have

$$
\left\langle[[u]]^{2}\right\rangle \leq 2\left\langle[[v]]^{2}\right\rangle+2 \int_{\Omega}\left|U^{\prime}\right|^{2} d x
$$

and then we use (3.8) to estimate the second term on the right hand side.

Our estimate (3.9) has the same form as the usual estimate in turbulence theory of the averaged energy dissipation rate for the Navier-Stokes boundary driven flow in a rectangular domain [3], [4], [6], [10].

In section 5 we shall use the estimates of $\epsilon$ to find an upper bound of the dimension of the global attractor.

4. A new version of the Lieb-Thirring inequality. In the present study we prove a generalization of the form of the Lieb-Thirring inequality obtained in [10] for a rectangular domain.

Let

$$
\tilde{H}^{1}=\left\{v \in \mathcal{C}^{\infty}\left(\Omega_{\infty}\right)^{2}: v \text { is } L \text {-periodic in } x_{1}, v_{\left.\right|_{\Gamma_{0}}}=0, v \cdot n_{\left.\right|_{\Gamma_{1}}}=0\right\}
$$

and

$$
H^{1}=\text { closure of } \tilde{H}^{1} \text { in } H^{1}(\Omega) \times H^{1}(\Omega) .
$$

Lemma 4.1. Let $\varphi_{j} \in H^{1}, j=1, \ldots, m$ be a suborthonormal family in $L^{2}(\Omega)$. Then

$$
\int_{\Omega}\left(\sum_{j=1}^{m} \varphi_{j}^{2}\right)^{2} \leq \sigma_{1} \sum_{j=1}^{m} \int_{\Omega}\left|\nabla \varphi_{j}\right|^{2}+\sigma_{2} m+\sigma_{3},
$$

where $\sigma_{1}=\kappa_{1}\left(1+\max _{0 \leq x_{1} \leq L}\left|h^{\prime}\left(x_{1}\right)\right|^{2}\right), \sigma_{2}=\kappa_{2}\left(\frac{1}{L^{2}}+\frac{1}{h_{0}^{2}}\right)$,

$$
\sigma_{3}=\kappa_{3} \int_{\Omega}\left(\frac{h^{\prime}\left(x_{1}\right)}{h\left(x_{1}\right)}\right)^{4}\left(1+h^{\prime}\left(x_{1}\right)^{4}\right) d x,
$$

and $\kappa_{1}, \kappa_{2}, \kappa_{3}$ are some absolute constants.

Proof. Let $\Omega_{1}=(0, L) \times\left(0, h_{0}\right)$, and let $\psi_{j} \in H^{1}\left(\Omega_{1}\right), j=1, \ldots, m$, be a family of functions that are suborthonormal in $L^{2}\left(\Omega_{1}\right)$ in the sense that

$$
\sum_{i, j=1}^{m} \xi_{i} \xi_{j} \int_{\Omega_{1}} \psi_{i} \psi_{j} d y \leq \sum_{k=1}^{m} \xi_{k}^{2}, \quad \forall \xi \in \mathbb{R}^{m} .
$$

We know ([10], Lemma 4.1) that for this family there exists an absolute constant $C_{0}$, such that

$$
\begin{aligned}
\int_{\Omega_{1}}\left(\sum_{j=1}^{m} \psi_{j}^{2}\right)^{2} d y \leq & C_{0}\left(\sum_{j=1}^{m} \int_{\Omega_{1}}\left(\frac{\partial \psi_{j}}{\partial y_{1}}\right)^{2} d y+\frac{\left|\psi_{j}\right|_{L^{2}\left(\Omega_{1}\right)}^{2}}{L^{2}}\right)^{\frac{1}{2}} \\
& \times\left(\sum_{j=1}^{m} \int_{\Omega_{1}}\left(\frac{\partial \psi_{j}}{\partial y_{2}}\right)^{2} d y+\frac{\left|\psi_{j}\right|_{L^{2}\left(\Omega_{1}\right)}^{2}}{h_{0}^{2}}\right)^{\frac{1}{2}},
\end{aligned}
$$

Now, for our family $\varphi_{j}$ defined in $\Omega$, we set

$$
\psi_{j}\left(y_{1}, y_{2}\right)=\varphi_{j}\left(x_{1}, x_{2}\right) \sqrt{\frac{h\left(x_{1}\right)}{h_{0}}}
$$


where

$$
h_{0}=\min _{0 \leq x_{1} \leq L} h\left(x_{1}\right), \quad y_{1}=x_{1}, \quad y_{2}=x_{2} \frac{h_{0}}{h\left(x_{1}\right)} .
$$

For $x=\left(x_{1}, x_{2}\right)$ in $\Omega, y=\left(y_{1}, y_{2}\right)$ is in $\Omega_{1}$, and the family $\psi_{j}, j=1, \ldots, m$, in $\Omega_{1}$ has the claimed properties. Changing variables in the above inequality and observing that

$$
\begin{aligned}
& d y_{1} d y_{2}=\frac{h_{0}}{h\left(x_{1}\right)} d x_{1} d x_{2} \\
& \frac{\partial \psi_{j}}{\partial y_{1}}=\left(\frac{\partial \varphi_{j}}{\partial x_{1}} \sqrt{\frac{h\left(x_{1}\right)}{h_{0}}}+\varphi_{j} \frac{h^{\prime}\left(x_{1}\right)}{2 \sqrt{h_{0} h\left(x_{1}\right)}}\right)+\sqrt{\frac{h\left(x_{1}\right)}{h_{0}}} \frac{\partial \varphi_{j}}{\partial x_{2}} \frac{h^{\prime}\left(x_{1}\right)}{h\left(x_{1}\right)} x_{2}, \\
& \frac{\partial \psi_{j}}{\partial y_{2}}=\frac{\partial \varphi_{j}}{\partial x_{2}} \sqrt{\frac{h\left(x_{1}\right)}{h_{0}}}
\end{aligned}
$$

with $h\left(x_{1}\right) / h_{0} \geq 1$, we obtain

$$
\begin{aligned}
\int_{\Omega}\left(\sum_{j=1}^{m} \varphi_{j}^{2}\right)^{2} d x \leq C_{0}\left(\sum _ { j = 1 } ^ { m } \int _ { \Omega } \left(\frac{\partial \varphi_{j}}{\partial x_{1}} a\right.\right. & \left.\left.+\varphi_{j} b+a \mu \frac{\partial \varphi_{j}}{\partial x_{2}} x_{2}\right)^{2} \frac{d x}{a^{2}}+\frac{\left|\varphi_{j}\right|_{L^{2}(\Omega)}^{2}}{L^{2}}\right)^{\frac{1}{2}} \\
& \times\left(\sum_{j=1}^{m} \int_{\Omega}\left(\frac{\partial \varphi_{j}}{\partial x_{2}}\right)^{2} d x+\frac{\left|\varphi_{j}\right|_{L^{2}(\Omega)}^{2}}{h_{0}^{2}}\right)^{\frac{1}{2}}
\end{aligned}
$$

where

$$
a=\sqrt{\frac{h\left(x_{1}\right)}{h_{0}}}, \quad b=\frac{h^{\prime}\left(x_{1}\right)}{2 \sqrt{h_{0} h\left(x_{1}\right)}}, \quad \mu=\frac{h^{\prime}\left(x_{1}\right)}{h\left(x_{1}\right)} .
$$

After simple calculations we get

$$
\begin{gathered}
\int_{\Omega}\left(\sum_{j=1}^{m}\left(\varphi_{j}\right)^{2}\right)^{2} d x \leq \frac{C_{0}}{2} \sum_{j=1}^{m} \int_{\Omega}\left(\left(\frac{\partial \varphi_{j}}{\partial x_{1}}\right)^{2}+\left(\frac{\partial \varphi_{j}}{\partial x_{2}}\right)^{2}\right) d x+C_{0}\left|\varphi_{j}\right|_{L^{2}(\Omega)}^{2}\left(\frac{1}{L^{2}}+\frac{1}{h_{0}^{2}}\right) \\
+\frac{C_{0}}{2} \int_{\Omega} \sum_{j=1}^{m} \frac{\partial \varphi_{j}}{\partial x_{1}} \varphi_{j} \mu d x+\frac{C_{0}}{8} \int_{\Omega}\left(\sum_{j=1}^{m} \varphi_{j}^{2}\right) \mu^{2} d x+C_{0} \int_{\Omega} \sum_{j=1}^{m} \frac{\partial \varphi_{j}}{\partial x_{1}} \frac{\partial \varphi_{j}}{\partial x_{2}} \mu x_{2} d x \\
+\frac{C_{0}}{2} \int_{\Omega} \sum_{j=1}^{m} \varphi_{j} \frac{\partial \varphi_{j}}{\partial x_{2}} \mu^{2} x_{2} d x+\frac{C_{0}}{2} \int_{\Omega} \sum_{j=1}^{m}\left(\frac{\partial \varphi_{j}}{\partial x_{2}}\right)^{2} \mu^{2} x_{2}^{2} d x
\end{gathered}
$$

When $h^{\prime}=0$, only the first two terms on the right hand side are not zero. We estimate the additional terms as follows.

$$
\begin{array}{r}
\frac{C_{0}}{2} \int_{\Omega} \sum_{j=1}^{m} \frac{\partial \varphi_{j}}{\partial x_{1}} \varphi_{j} \mu d x \leq \frac{C_{0}}{2} \int_{\Omega}\left(\sum_{j=1}^{m}\left(\frac{\partial \varphi_{j}}{\partial x_{1}}\right)^{2}\right)^{\frac{1}{2}}\left(\sum_{j=1}^{m} \varphi_{j}^{2}\right)^{\frac{1}{2}} \mu d x \\
\leq \frac{C_{0}}{2} \int_{\Omega} \sum_{j=1}^{m}\left(\frac{\partial \varphi_{j}}{\partial x_{1}}\right)^{2} d x+\frac{C_{0}}{8} \int_{\Omega}\left(\sum_{j=1}^{m} \varphi_{j}^{2}\right) \mu^{2} d x \\
\leq \frac{C_{0}}{2} \int_{\Omega} \sum_{j=1}^{m}\left(\frac{\partial \varphi_{j}}{\partial x_{1}}\right)^{2} d x+\frac{1}{16} \int_{\Omega}\left(\sum_{j=1}^{m} \varphi_{j}^{2}\right)^{2} d x+\frac{\left(C_{0}\right)^{2}}{16} \int_{\Omega} \mu^{4}\left(x_{1}\right) d x
\end{array}
$$


and

$$
\frac{C_{0}}{8} \int_{\Omega}\left(\sum_{j=1}^{m} \varphi_{j}^{2}\right) \mu^{2} d x \leq \frac{1}{16} \int_{\Omega}\left(\sum_{j=1}^{m} \varphi_{j}^{2}\right)^{2} d x+\frac{\left(C_{0}^{\prime}\right)^{2}}{16} \int_{\Omega} \mu^{4}\left(x_{1}\right) d x
$$

Now,

$$
\begin{array}{r}
C_{0} \sum_{\Omega} \sum_{j=1}^{m} \frac{\partial \varphi_{j}}{\partial x_{1}} \frac{\partial \varphi_{j}}{\partial x_{2}} \mu x_{2} d x \leq C_{0} \int_{\Omega}\left(\sum_{j=1}^{m}\left(\frac{\partial \varphi_{j}}{\partial x_{1}}\right)^{2}\right)^{\frac{1}{2}}\left(\sum_{j=1}^{m}\left(\frac{\partial \varphi_{j}}{\partial x_{2}}\right)^{2}\right)^{\frac{1}{2}} \mu x_{2} d x \\
\leq \frac{C_{0}}{2} \int_{\Omega} \mu^{2}\left(x_{1}\right) x_{2}^{2} \sum_{j=1}^{m}\left(\frac{\partial \varphi_{j}}{\partial x_{1}}\right)^{2} d x+\frac{C_{0}}{2} \int_{\Omega} \sum_{j=1}^{m}\left(\frac{\partial \varphi_{j}}{\partial x_{2}}\right)^{2} d x
\end{array}
$$

and

$$
\begin{array}{r}
\frac{C_{0}}{2} \int_{\Omega} \sum_{j=1}^{m} \varphi_{j} \frac{\partial \varphi_{j}}{\partial x_{2}} \mu^{2} x_{2} d x \leq \frac{C_{0}}{2} \int_{\Omega}\left(\sum_{j=1}^{m} \varphi_{j}^{2}\right)^{\frac{1}{2}}\left(\sum_{j=1}^{m}\left(\frac{\partial \varphi_{j}}{\partial x_{2}}\right)^{2}\right)^{\frac{1}{2}} \mu^{2} x_{2} d x \\
\leq \frac{C_{0}}{8} \int_{\Omega}\left(\sum_{j=1}^{m} \varphi_{j}^{2}\right) \mu^{4} x_{2}^{2} d x+\frac{C_{0}}{2} \int_{\Omega} \sum_{j=1}^{m}\left(\frac{\partial \varphi_{j}}{\partial x_{2}}\right)^{2} d x \\
\leq \frac{1}{16} \int_{\Omega}\left(\sum_{j=1}^{m} \varphi_{j}^{2}\right)^{2} d x+\frac{\left(C_{0}\right)^{2}}{16} \int_{\Omega} \mu^{8}\left(x_{1}\right) x_{2}^{4} d x+\frac{C_{0}}{2} \int_{\Omega} \sum_{j=1}^{m}\left(\frac{\partial \varphi_{j}}{\partial x_{2}}\right)^{2} d x .
\end{array}
$$

Applying the above inequalities in (4.4) and replacing $x_{2}$ by $h\left(x_{1}\right)$ in some integrals we obtain the elegant estimate

$$
\begin{array}{r}
\int_{\Omega}\left(\sum_{j=1}^{m} \varphi_{j}^{2}\right)^{2} \leq C_{0}^{\prime \prime} \sum_{j=1}^{m} \int_{\Omega}\left(1+h^{\prime}\left(x_{1}\right)^{2}\right)\left|\nabla \varphi_{j}\right|^{2}+C_{0}\left(\frac{1}{L^{2}}+\frac{1}{h_{0}^{2}}\right) \sum_{j=1}^{m}\left|\varphi_{j}\right|_{L^{2}(\Omega)} \\
+C_{3}^{\prime \prime} \int_{\Omega}\left(\frac{h^{\prime}\left(x_{1}\right)}{h\left(x_{1}\right)}\right)^{4}\left(1+h^{\prime}\left(x_{1}\right)^{4}\right) d x
\end{array}
$$

whence, as

$$
\sum_{j=1}^{m}\left|\varphi_{j}\right|_{L^{2}(\Omega)}=m
$$

(4.1) follows.

5. Dimension estimate of the global attractor. We rewrite equation (2.13) for the homogenized flow $v$ in the short form

$$
\frac{d v}{d t}=L(v), \quad v(0)=v_{0},
$$

where, for all $\Theta \in V$,

$$
\langle L(v(t)), \Theta\rangle=-a(v(t), \Theta)-B(v(t), v(t), \Theta)+F(v(t), \Theta)
$$

and $F$ is defined in (2.14). Now, to estimate from above the dimension of the global attractor we follow the standard procedure, cf. e.g. [7], [8]. First, for an integer $m>1$ and vectors $\xi_{j} \in H, j=1, \ldots, m$, we consider the corresponding problems linearized 
about the orbit $v(t)$,

$$
\frac{d}{d t} U_{j}=L^{\prime}(v) U_{j}, \quad U_{j}(0)=\xi_{j}, \quad \text { for } \quad j=1, \ldots, m
$$

where $L^{\prime}(v)$ is the Fréchet derivative of $L$ at $v=v(t)$, with

$$
\begin{aligned}
\left(L^{\prime}(v) U_{j}, \Theta\right)=-a\left(U_{j}, \Theta\right) & -B\left(v, U_{j}, \Theta\right)-B\left(U_{j}, v, \Theta\right) \\
& -B\left(\xi, U_{j}, \Theta\right)-B\left(U_{j}, \xi, \Theta\right) \\
& =-a\left(U_{j}, \Theta\right)-B\left(u, U_{j}, \Theta\right)-B\left(U_{j}, u, \Theta\right) .
\end{aligned}
$$

Let, for a particular time $\tau, \Theta_{j}=\Theta_{j}(\tau), j \in N$ be an orthonormal basis of $H$ with $\Theta_{1}(\tau), \ldots, \Theta_{m}(\tau)$ spanning

$$
Q_{m}(\tau) H=Q_{m}\left(\tau, v_{0}, \xi_{1}, \ldots, \xi_{m}\right) H=\operatorname{Span}\left[U_{1}(\tau), \ldots, U_{m}(\tau)\right]
$$

$Q_{m}(\tau)$ being the orthogonal projector of $H$ onto the space spanned by $U_{j}(\tau), j=$ $1, \ldots, m$, solutions of $(5.1)$. We then have $\Theta_{j}(\tau) \in V, j=1, \ldots, m$, for a.e. $\tau \in \mathbb{R}_{+}$.

The trace of $L^{\prime}(v(\tau)) \circ Q_{m}(\tau)$ is

$$
\operatorname{Tr}\left(L^{\prime}(v(\tau)) \circ Q_{m}(\tau)\right)=\sum_{j=1}^{m}\left(L^{\prime}(v(\tau)) \Theta_{j}(\tau), \Theta_{j}(\tau)\right) .
$$

Let $\mathcal{A}$ be the global attractor for the homogenized flow $v(\tau)=\tilde{S}(\tau) v_{0}$, and let

$$
q_{m}=\lim _{T \rightarrow \infty} \sup q_{m}(T),
$$

where

$$
q_{m}(T)=\sup _{v_{0} \in \mathcal{A}} \sup \left\{\frac{1}{T} \int_{0}^{T} \operatorname{Tr}\left(L^{\prime}(v(\tau)) \circ Q_{m}(\tau)\right) d \tau: \xi_{j} \in H,\left[\xi_{j}\right] \leq 1, j=1, \ldots, m\right\} .
$$

Then, the fractal dimension of the attractor $\mathcal{A}$ is less than or equal to the first nonnegative integer $m_{0}$ for which $q_{m_{0}} \leq 0$, [7].

Now, our aim is to obtain estimate (5.7) and then inequality (5.8).

LEMMA 5.1. The following estimate holds:

$$
\operatorname{Tr}\left(L^{\prime}(v) \circ Q_{m}\right) \leq-\nu \sum_{j=1}^{m}\left[\left[\Theta_{j}\right]\right]^{2}+|\rho|_{L^{2}(\Omega)}[[u]]
$$

where

$$
\rho(x)=\sum_{j=1}^{m}\left|\Theta_{j}(x)\right|^{2}
$$

Proof. From (5.2) and (5.3) we obtain

$$
\begin{array}{r}
\sum_{j=1}^{m}\left(L^{\prime}(v) \Theta_{j}, \Theta_{j}\right) \leq-\nu \sum_{j=1}^{m}\left[\left[\Theta_{j}\right]\right]^{2}-\sum_{j=1}^{m} B\left(\Theta_{j}, u, \Theta_{j}\right) \\
\leq-\nu \sum_{j=1}^{m}\left[\left[\Theta_{j}\right]\right]^{2}+\int_{\Omega}|\nabla u|\left(\sum_{j=1}^{m}\left|\Theta_{j}(x)\right|^{2}\right) d x
\end{array}
$$

whence (5.4) follows. 
Now, in our estimates of the trace we use lemma 4.1. By this lemma and from

$$
\int_{\Omega} \rho(x) d x=m
$$

we have

$$
\frac{m^{2}}{|\Omega|} \leq|\rho|_{L^{2}(\Omega)}^{2} \leq \sigma_{1} \sum_{j=1}^{m}\left[\left[\Theta_{j}\right]\right]^{2}+\sigma_{2} m+\sigma_{3}
$$

Moreover,

$$
|\rho|_{L^{2}(\Omega)}[[u]] \leq \frac{\nu}{2 \sigma_{1}}|\rho|_{L^{2}(\Omega)}^{2}+\frac{\sigma_{1}}{2 \nu}[[u]]^{2} \leq \frac{\nu}{2}\left(\sum_{j=1}^{m}\left[\left[\Theta_{j}\right]\right]^{2}+\frac{\sigma_{2}}{\sigma_{1}} m+\frac{\sigma_{3}}{\sigma_{1}}\right)+\frac{\sigma_{1}}{2 \nu}[[u]]^{2}
$$

by the second inequality in (5.5). Thus, by (5.4), (5.6),

$$
\operatorname{Tr}\left(L^{\prime}(v) \circ Q_{m}\right) \leq \frac{-\nu}{2} \sum_{j=1}^{m}\left[\left[\Theta_{j}\right]\right]^{2}+\frac{\nu \sigma_{2}}{2 \sigma_{1}} m+\frac{\nu \sigma_{3}}{2 \sigma_{1}}+\frac{\sigma_{1}}{2 \nu}[[u]]^{2},
$$

and by (5.5) again, we fianally obtain

$$
\operatorname{Tr}\left(L^{\prime}(v(\tau)) \circ Q_{m}(\tau)\right) \leq \frac{-\nu}{2 \sigma_{1}|\Omega|} m^{2}+\frac{\nu \sigma_{2}}{\sigma_{1}} m+\frac{\nu \sigma_{3}}{\sigma_{1}}+\frac{\sigma_{1}}{2 \nu}[[u]]^{2} .
$$

From (5.7), recalling $\epsilon$ defined in (3.1), we have

$$
q_{m} \leq \frac{-\nu}{2 \sigma_{1}|\Omega|} m^{2}+\frac{\nu \sigma_{2}}{\sigma_{1}} m+\frac{\nu \sigma_{3}}{\sigma_{1}}+\frac{\sigma_{1}|\Omega|}{2 \nu^{2}} \epsilon .
$$

Define

$$
a=\frac{\nu}{2 \sigma_{1}|\Omega|}, \quad 2 b=\frac{\nu \sigma_{2}}{\sigma_{1}}, \quad c=\frac{\nu \sigma_{3}}{\sigma_{1}}+\frac{\sigma_{1}|\Omega|}{2 \nu^{2}} \epsilon .
$$

We can write

$$
q_{m} \leq-a m^{2}+2 b m+c
$$

Let

$$
p=\frac{b+\sqrt{b^{2}+a c}}{a}
$$

and let $E(p)$ denote the integer part of $p$. We have $m_{0}=E(p)+1$ if $p \neq E(p)$, and $m_{0}=p$ if $p=E(p)$. Using the inequality $\sqrt{x^{2}+y^{2}} \leq \sqrt{2} \max \{|x|,|y|\}$ we can estimate $p$ by a simpler expression,

$$
p \leq 3 \max \left\{\frac{b}{a}, \sqrt{\frac{c}{a}}\right\} .
$$

Further, taking into account definitions of $a, b, c$, as well as estimate (3.9) of $\epsilon$, we obtain

$$
p \leq 3 \max \left\{\sigma_{2}|\Omega|, \sqrt{2 \sigma_{3}|\Omega|+\sigma_{1}^{2} \frac{|\Omega|^{2}}{h_{0}^{4}}(R e)^{3}}\right\} .
$$

From the above estimates we deduce the following theorem about strongly turbulent flows in thin domains. 
THEOREM 5.1. Consider the Navier-Stokes flow $u$ as described in section 2. Assume that the domain $\Omega$ is thin and that the flow is strongly turbulent, namely

$$
\frac{h_{M}}{L}<<1 \text { and } \quad \text { Re }>>1 .
$$

Then the fractal dimension of the global attractor $\mathcal{A}_{N S E}$ for this flow can be estimated as follows:

$$
\operatorname{dim}\left(\mathcal{A}_{N S E}\right) \leq \kappa \max \left\{\sigma_{2}|\Omega|, \sqrt{2 \sigma_{3}|\Omega|+\sigma_{1}^{2}\left(\frac{L h_{M}}{h_{0}^{2}}\right)^{2}(R e)^{3}} .\right.
$$

where $\sigma_{1}=\kappa_{1}\left(1+\max _{0 \leq x_{1} \leq L}\left|h^{\prime}\left(x_{1}\right)\right|^{2}\right), \sigma_{2}|\Omega|:=\kappa_{2}\left(\frac{1}{L^{2}}+\frac{1}{h_{0}^{2}}\right)|\Omega|$,

$$
\sigma_{3}=\kappa_{3} \int_{\Omega}\left(\frac{h^{\prime}\left(x_{1}\right)}{h\left(x_{1}\right)}\right)^{4}\left(1+h^{\prime}\left(x_{1}\right)^{4}\right) d x,
$$

and where $\kappa, \kappa_{1}$, and $\kappa_{3}$ are some numeric constants. For a rectangular domain $\Omega=$ $(0, L) \times\left(0, h_{0}\right)$ we obtain, in particular,

$$
\operatorname{dim}\left(\mathcal{A}_{N S E}\right) \leq \kappa \frac{L}{h_{0}}(R e)^{3 / 2} .
$$

Proof. By (5.9), (5.11) follows.

Estimate (5.12) was obtained in [5] for the case of homogeneous boundary condition on the top part of the boundary. Estimate (5.11) is its direct generalization for more general geometry of the flow domain. Observe that the estimate depends explicitly on the first derivative $h^{\prime}$.

6. Conclusions. In section 3 we estimated the time averaged energy dissipation rate of the considered flow applying the background flow method. We used a version of Hopf's construction of the background flow and obtained the same estimate as that obtained earlier for a rectangular domain by Doering and Constantin who used a background flow suitable for the channel case, cf. [3], [4].

In section 4 we generalized an anisotropic version of the Lieb-Thirring inequality obtained in [10], from rectangular to non-rectangular domains defined in section 2 . This inequality can be generalized further to include three dimensional domains.

In section 5 we provided an estimate of the dimension of the global attractor of the considered flow, with constants given explicitly in terms of the data, including geometry of the domain. In particular, for a rectangle, our estimate reduces to that obtained earlier, cf. e.g. [5]. Inequality (5.11) agrees with our expectations about the behavior of strongly turbulent shear flows in thin domains met in lubrication theory. It helps to understand the influence of geometry of the flow and roughness of the boundary on the fluid behavior. Moreover, inequality (5.9) serves as an estimate of the number of degrees of freedom of turbulent flows not only in thin films.

Acknowledgements. Partial support from the EC financed network HYKE no. HPRNCT-2002-00282 is gratefully acknowledged. 


\section{References}

[1] M. Boukrouche and G. Eukaszewicz, An upper bound on attractor dimension of a $2 D$ turbulent shear flows in lubrication theory, Nonlinear Analysis 59 (2004), 1077-1089.

[2] M. Boukrouche and G. Eukaszewicz, Attractor dimension estimate for plane shear flow of micropolar fluid with free boundarys, Math. Methods Appl. Sci. 28 (2005), 1673-1694.

[3] Ch. R. Doering and P. Constantin, Energy dissipation in shear driven turbulence, Phys. Rev. Lett. 69 (1991), 1648-1651.

[4] Ch. R. Doering and J. D. Gibbon, Applied Analysis of the Navier-Stokes Equations, Cambridge University Press, Cambridge, 1995.

[5] Ch. R. Doering and X. Wang, Attractor dimension estimates for two-dimensional shear flows, Physica D 123 (1998), 206-222.

[6] C. Foias, O. Manley, R. Rosa and R. Temam, Navier-Stokes Equations and Turbulence, Cambridge University Press, Cambridge, 2001.

[7] J. C. Robinson, Infinite Dimensional Dynamical Systems, Cambridge University Press, Cambridge, 2001.

[8] R. Temam, Infinite Dimensional Dynamical Systems in Mechanics and Physics, 2nd ed., Springer-Verlag, New York, 1997.

[9] R. Temam and M. Ziane, Navier-Stokes equations in three-dimensional thin domains with various boundary conditions, Adv. in Differential Equations 1 (1998), 1-21.

[10] M. Ziane, On the 2D-Navier-Stokes equations with the free boundary condition, Appl. Math. and Optimization 38 (1998), 1-19. 